This is an open access article distributed under the terms of the Creative Commons BY-NC-ND Licence

\title{
Identification and validation of reference genes for real-time qPCR normalization during Al-induced programmed cell death in peanut
}

\author{
S.C. YAO ${ }^{123}$, J. ZHAN ${ }^{1}$, C.L. PAN ${ }^{13}$, W.J. XIONG ${ }^{1}$, D. XIAO ${ }^{1}$, Y.L. WANG ${ }^{1}$, H. $\mathrm{SHEN}^{4}$, A.Q. WANG ${ }^{1 *}$, \\ and L.F. HE ${ }^{1 *}$
}

College of Agronomy, Guangxi University, Nanning, 530005, P.R. China ${ }^{1}$

Institute of Marine Drugs, Guangxi University of Chinese Medicine, Nanning, 530001, P.R. China ${ }^{2}$

College of Life Science and Technology, Guangxi University, Nanning, 530005, P.R. China ${ }^{3}$

College of Agriculture, South China Agricultural University, Guangzhou, 510642, P.R. China ${ }^{4}$

\begin{abstract}
The reverse transcription quantitative real-time PCR (RT-qPCR) is becoming increasingly important for gene expression studies. However, the accuracy and reliability of RT-qPCR depend on normalizing expression to reference genes. In this study, ten candidate reference genes, including cyclophilin (CYP), elongation factor $1 b(E F 1 b), \alpha$-tubulin (TUA5), B-tubulin (TUB4), ubiquitin10R (UBQ10R), 60S ribosomal RNA (60S), alcohol dehydrogenase (ADH3), metalloprotease (MTP), glyceraldehyde-3-phosphate dehydrogenase (GAPDH), and actin (ACT2) were evaluated for the stability of expression in three tissues of two peanut cultivars [Zhonghua $2\left(\mathrm{ZH}_{2}\right)$ and 99-1507] under Al stress by four statistical algorithms (geNorm, NormFinder, BestKeeper, and RefFinder). The results suggested that the top-ranked reference genes under Al-induced programmed cell death (PCD) in peanut were UBQ10R, EF1b and CYP, with the most suitable combination of reference genes being [UBQ10R+ACT2]. The UBQ10R exhibited the most stable expression in all samples, while TUB4 was the least stable gene. The relative expression of $A h M C 1$ (the caspase-like protease family gene, which played a significant role in Al-induced PCD) showed that there was no significant difference with the best reference gene and the best gene combination in RT-qPCR normalization, but there was significant difference with the least stable gene TUB4 as reference gene. This is the first study to evaluate the stability of reference genes in peanut under Al-induced PCD, and the results will provide guidance to identify appropriate reference genes for further RT-qPCR analyses under Al stress in peanut.
\end{abstract}

Additional key words: actin, Arachis hypogea, elongation factor 1b, glyceraldehyde-3-phosphate dehydrogenase, $\beta$-tubulin, ubiquitin.

\section{Introduction}

Gene expression profiles play an important role in molecular biology (VanGuilder et al. 2008). Several techniques have been used to reveal profiles of gene expression, such as Northern blotting, DNA microarray, in situ hybridization (ISH), gene chip, semi-quantitative (sq) and quantitative (q) PCR (Stephenson 2010). With the advantage of higher sensitivity, specificity, speed, accuracy, throughput, and lower cost, reverse transcription (RT)-qPCR has become the most popular method for the relative quantification of gene expression in recent years (Sugden and Winter 2008, Kulcheski et al. 2010, Li et al. 2012). However, the accuracy and reliability of RT-qPCR

Submitted 19 March 2018, last revision 18 September 2018, accepted 27 September 2018.

Abbreviations: Ac-DEVD-CHO - acetyl-Asp-Glu-Val-Asp-aldehyde; ACT - actin; BLAST - basic local alignment search tool; Cq - the threshold (or quantification) cycle; CV - coefficient of variation; DMSO - dimethyl sulfoxide; EF-1b - elongation factor 1b; GAPDH - glyceraldehyde-3-phosphate dehydrogenase; M - the average expression stability values; PCD - programmed cell death; RT-qPCR reverse transcription quantitative polymerase chain reaction; 18S - ribosomal RNA $18 S$; TUB - $\beta$-tubulin; UBQ - ubiquitin; V - the pairwise variation.

Acknowledgements: This work was supported by the National Natural Science Foundation of China (grant Nos. 31560346, 31660350 , 31776190 and 31701356), the Guangxi Natural Science Foundation of China (grant No. 2014GXNSFAA118074) and Innovation Project of Guangxi Graduate Education (grant No. YCSW2017042). The first two authors contributed equally to this work.

* Corresponding authors; e-mails: waiqing1966@126.com ;lfhe@gxu.edu.cn. 
is restricted by the amount of sample, quality of RNA, and normalization, which used reference genes (Van Guilder et al. 2008). Reference genes are usually recruited from the category of housekeeping genes, including actin $(A C T)$, glyceraldehyde-3-phosphate dehydrogenase (GAPDH), ubiquitin $(U B Q), \beta$-tubulin $(T U B)$, elongation factor $1 \mathrm{~b}$ (EF1b), and $18 S$ or $60 S$ ribosomal RNA (18S or $60 S)$ (Kim et al. 2003). Appropriate reference genes in RT-qPCR should ideally exhibit constitutive expression in various tissues, at different developmental stages, or under different experimental conditions (Wan and Wilkins 1994, Nolan et al. 2006, Xiang et al. 2008, Zhong et al. 2011). However, recent studies have shown that no single reference gene exhibited constant expression under all experimental conditions (Mehdi and Van 2012, Galeano et al. 2014, Ling et al. 2014). Therefore, re-evaluation of a suitable reference gene or multiple stably-expressed reference genes can improve the accuracy of RT-qPCR system, reduce inaccurate data interpretation, and reflect better the actual expressions of target genes.

The expression stability of candidate reference genes was evaluated by different algorithms, estimating the stability parameter in each, in order to identify the most appropriate reference gene from different candidates. Several widely-used statistical algorithm packages (BestKeeper, geNorm, Normfinder, and RefFinder) have been recommended for reference gene evaluation in recent years (Patankar et al. 2016, Freitas et al. 2017, Hou et al. 2017).

Peanut (Arachis hypogaea L.) is an important crop throughout the world, which is generally cultivated in acid soil in southern China. Aluminum is uptaken from the soil as $\mathrm{Al}^{3+}$ once the $\mathrm{pH}$ falls below 5.0 (Pan et al. 2001). Our previous studies have shown that $\mathrm{Al}$ rapidly induced mitochondria-dependent programmed cell death (PCD) in peanut, and there was a negative relationship between Al-induced PCD and Al tolerance (Zhan et al. 2013, 2014,

\section{Materials and methods}

Plants and treatments: Peanut (Arachis hypogea L.) cultivars Zhonghua $2\left(\mathrm{ZH}_{2} ; \mathrm{Al}\right.$-sensitive) and 99-1507 (Al-tolerant) were chosen to carry out all the experiments. Plants were cultivated as described by Yao et al. (2016). Some seedlings at 3-leaf stage were treated with $100 \mu \mathrm{M}$ $\mathrm{AlCl}_{3}$ in Hoagland nutrient solution ( $\mathrm{pH}$ 4.2) for five different times $(0,4,8,12$, and $24 \mathrm{~h})$ after pretreatment with $0.1 \mathrm{mM} \mathrm{CaCl}_{2}$ solution $(\mathrm{pH} 4.5)$ for $1 \mathrm{~d}$. The other seedlings were treated with the caspase-3-like inhibitor acetyl-Asp-Glu-Val-Asp-aldehyde (Ac-DEVD-CHO) [50 $\mu \mathrm{M}$ dissolved in dimethyl sulfoxide (DMSO), $\mathrm{pH} 4.5$ ] which can inhibit Al-induced PCD (Jiao et al. 2013). Four treatments of Ac-DEVD-CHO were performed: $50 \mu \mathrm{M}$ DMSO alone (control), $50 \mu \mathrm{M}$ Ac-DEVD-CHO alone (DEVD), $100 \mu \mathrm{M} \mathrm{Al}$ alone (Al), and $100 \mu \mathrm{M} \mathrm{Al}+50 \mu \mathrm{M}$ Ac-DEVD-CHO (Al+DEVD). To achieve the maximum
Huang et al. 2014b). However, the mechanism of Al-induced PCD is still unclear. With the development of high-throughput sequencing technology, more and more genomes have been successfully sequenced, and the expression of genes related to $\mathrm{Al}$ tolerance have been studied. This requires an efficient tool, such as RT-qPCR, to analyze the expression profile of Al-responsive genes, to dissect the mechanism of Al-induced PCD.

In recent years, some progresses have been made with respect to reference gene normalization for RT-qPCR in peanut, demonstrating that expression of reference genes changed considerably in different developmental stages and in different experimental conditions. Yael and Ran (2010) suggested that the most stable reference gene is alcohol dehydrogenase (ADH3), followed by $60 S$ and $y l s 8$ in various developmental stages of peanut. Reddy et al. (2013) demonstrated that $A D H 3$ and glucose-6phospahate 1-dehydrogenase (G6PD) were stably expressed across experiments, while EFlb might potentially be better over a diverse set of tissue samples of peanut when subjected to various biotic (virus infection) and abiotic (salinity and drought) stresses. Morgante et al. (2011) found that $A C T 1, U B I 1$ and GAPDH are the most stable genes for different species, organs, and treatments. To date, appropriate reference genes have not yet been validated for Al-induced PCD in peanut.

The aim of present study was to evaluate the expressions of ten candidate housekeeping genes including cyclophilin (CYP), EF1b, $\alpha$-tubulin (TUA5), TUB4, UBQ10R, 60S, ADH3, metalloprotease (MTP), GAPDH and $A C T 2$ in two peanut cultivars subjected to abiotic stresses (Al and caspase-3-like inhibitor Ac-DEVD-CHO) and in three different tissues and to compare expression stability by GeNorm (Vandesompele et al. 2002), NormFinder (Andersen et al. 2004), BestKeeper (Pfaffl et al. 2004), and RefFinder (Xie et al. 2011) programs.

inhibitory effect, Ac-DEVD-CHO was applied $2 \mathrm{~h}$ prior to adding $100 \mu \mathrm{M} \mathrm{Al}$ for $8 \mathrm{~h}$. Ten seedlings per replicate and three replicates of each treatment were done. The different tissue samples including root tips, stems, and leaves were collected, snap-frozen in liquid nitrogen, and stored at $-80^{\circ} \mathrm{C}$ for RNA extraction.

RNA isolation and cDNA synthesis: Total RNA was extracted from all samples with the Promega Eastep ${ }^{\circledR}$ super RNA extraction kit (Promega, Shanghai, China) according to the manufacturer's instructions. After extraction, the concentration of RNA was quantified with NanoDrop 2000c spectrophotometer (Thermo Scientific, Waltham, MA, USA). Only those RNA preparations, which satisfied the requirement for an $\mathrm{A}_{260} / \mathrm{A}_{280}$ ratio of 1.9 to 2.1 and an $A_{260} / A_{230}$ ratio greater than 2.0, were used 
for further analysis. The total RNA was diluted to $500 \mu \mathrm{g} \mathrm{cm}^{-3}$ and checked by $1 \%(\mathrm{~m} / \mathrm{v})$ agarose gel electrophoresis (Fig. 1. Suppl.). cDNA was synthesized using Prime Script ${ }^{\mathrm{TM}}$ RT reagent kit with gDNA eraser (Perfect Real Time) kit (Takara, Dalian, China) according to the manufacturer's instructions. Genomic DNA was cleanedup using the gDNA eraser procedure before the reverse transcription. The cDNA mixture was stored at $-20{ }^{\circ} \mathrm{C}$.

Selection of reference genes and primer design: Ten candidate reference genes (CYP, EF1b, TUA5, TUB4, $U B Q 10 R, 60 S, G A P D H, A C T 2, A D H 3, M T P$ ) involved in different functional classes in the cell were selected based on the previous description (Morgante et al. 2011, Chi et al. 2012, Reddy et al. 2013). They were used to BLAST (http://ncbi.nlm.nih.gov/blast) the peanut EST to obtain the corresponding homologous gene sequences in the genome. The primers were designed using Primer Premier 5.0 software (Premier, Toronto, Canada) and referred to previous reports in peanut (Yael and Ran 2010, Chen et al. 2011, Morgante et al. 2011, Chi et al. 2012, Reddy et al. 2013), with melting temperatures of $58-60{ }^{\circ} \mathrm{C}$, primer lengths of 18 - $25 \mathrm{bp}$ and amplicon lengths of $64-190 \mathrm{bp}$ (Table 1 Suppl.). Synthesis of all the primers was performed by Takara. The amplicons were analyzed by $1.0 \%$ agarose gel electrophoresis before RT-qPCR.

Real-time qPCR analysis for amplification specificity and efficiency: Real-time reactions were performed using the CFX 96TM real-time system (Bio-Rad, Hercules, CA, USA) in $20 \mathrm{~mm}^{3}$ of reaction mixture containing $10 \mathrm{~mm}^{3}$ of SYBR ${ }^{\circledR}$ Primix Ex Taq $^{\mathrm{TM}}$ (Bio-Rad), $2.0 \mathrm{~mm}^{3}$ of cDNA template, $1.0 \mathrm{~mm}^{3}$ of each primer $(10 \mathrm{mM})$ and $\mathrm{ddH}_{2} \mathrm{O}$; RT-qPCR reactions with no-template controls (NTC, containing $\mathrm{ddH}_{2} \mathrm{O}$ instead of cDNA) were also carried out for each primer pair to check for template contamination. All PCR reactions were performed under following conditions: an initial denaturation step of at $95{ }^{\circ} \mathrm{C}$ for $3 \mathrm{~min}$ and 40 cycles (each consisting of $10 \mathrm{~s}$ at $95^{\circ} \mathrm{C}, 30 \mathrm{~s}$ at $60{ }^{\circ} \mathrm{C}$, and $10 \mathrm{~s}$ at $95^{\circ} \mathrm{C}$ ), followed by melting curve analysis by heating from 65 to $95{ }^{\circ} \mathrm{C}$ with a speed of $0.5^{\circ} \mathrm{C}$ per $5 \mathrm{~s}$. A standard curve for every primer pair was carried out via a three-fold serial dilution of pooled cDNA. The amplification efficiency (E) was calculated using the formula $E=\left(2^{-1 / \text { slope }}-1\right) \times 100$. The slope value (regression coefficient) and the correlation coefficient $\left(r^{2}\right)$ were directly obtained from the equation of the line-of-best-fit of the standard curve.

Data handling and analysis: Expressions were usually estimated according to the number of amplification cycles taken to reach a fixed threshold, which is named the threshold (or quantification) cycle $(\mathrm{Cq})$ value. In the relative quantities method, $\mathrm{Cq}$ values are converted to $\Delta \mathrm{Cq}$ values and then compared. The smallest $\mathrm{Cq}$ is first defined as 1 and other $\mathrm{Cq}$ values subtracted from 1 to gain the $\Delta \mathrm{Cq}$ values. The smaller the $\Delta \mathrm{Cq}$, the more stable a candidate reference gene is.

The geNorm is based on the Excel-VBA applet platform which evaluates the stability of reference genes and recommends the number of reference genes. The principle of geNorm is that the expression ratio of two ideal reference genes should be the same in all the samples despite changes in experimental conditions or types of tissue used (Vandesompele et al. 2002). GeNorm calculates the average expression stability values (M) and the pairwise variation $(\mathrm{V})$ values for that gene, compared to the other genes. To execute, a $\Delta \mathrm{Cq}$ value was obtained and then imported into geNorm. There is a negative relationship between $M$ value and gene stability. The pairwise variation $\mathrm{V}_{\mathrm{n}} / \mathrm{V}_{\mathrm{n}+1}$ is also used in geNorm between two sequential normalization factors, $\mathrm{NF}_{\mathrm{n}}$ and $\mathrm{NF}_{\mathrm{n}+1}$, to determine the ideal number of reference genes. Once the $\mathrm{V}_{\mathrm{n}} / \mathrm{V}_{\mathrm{n}+1}$ ratio is below $0.15, \mathrm{n}$ is considered the suitable number of reference genes.

The NormFinder is also widely used to determine the expression stability of candidate reference genes (Andersen et al. 2004). The calculation of NormFinder is similar to geNorm, and $\Delta \mathrm{Cq}$ values are used. Intra- and inter-group variation among the sample subgroups are also estimated by this software, and the most stable one is identified.

The BestKeeper program calculates the correlation coefficient $(r)$, standard deviation (SD,) and coefficient of variation $(\mathrm{CV})$ by pairwise comparison of all the candidate genes. The genes with smaller SD values, possess greater stability. Reference genes with SD values $>1$ are considered to be the most unstable ones (Pfaffl et al. 2004).

For more reliable and consistent results, the RefFinder was applied to calculate the geometric mean of each candidate gene and to re-rank the stability (CastroQuezada et al. 2013, Zsóri et al. 2013). To execute, the ranking of each gene by geNorm, NormFinder and BestKeeper was multiplied by different weighting coefficients and calculated the geometric mean to produce an overall final ranking (Freitas et al. 2017).

Reference gene validation under Al-stress treatments: To determine how the most and the least stable reference genes affected gene expression level, a caspase-like protease gene $A h M C 1$ was chosen to be the target gene. AhMC1 plays an important role in Al-induced PCD in peanut and its ORF sequence has been successfully cloned from peanut (Yao et al., unpublished results). In addition, the most stable reference gene $U B Q 10 R$, and the most stable gene combination [UBQ10+ACT2], as well as the least stable reference gene $T U B 4$ were chosen as reference genes. The seedlings of $\mathrm{ZH}_{2}$ were treated with $100 \mu \mathrm{M} \mathrm{Al}$ for different times $(0,4,8,12$, and $24 \mathrm{~h})$ after pretreatment at $0.1 \mathrm{mM} \mathrm{CaCl}_{2}$ solution $(\mathrm{pH} 4.5)$ for $1 \mathrm{~d}$, and the root tips, stems, and leaves were collected for RT-qPCR. The primers (AhMC1-F: 5' TGACGATGATGGAAGTGCGG 3' 
and AhMCl-R: 5' CATAGCATTCAGCAAGCGGC 3') of AhMC1 were designed by Primer 5.0 software. The amplified length was sequenced, and specificity was confirmed by melt curve analysis. The relative expression of $A h M C 1$ was calculated by the $2^{-\Delta \Delta C q}$ formula (Kundu et al. 2013).

\section{Results}

A set of 42 pooled samples including three different tissues (roots, stems and leaves) of two peanut cultivars exposed to Al stress, was used to evaluate the expression stability of ten candidate genes for normalization of RT-qPCR. A single amplification of expected length was verified by $1 \%$ agarose gel electrophoresis, and a single peak was observed in the dissociation curve by RT-qPCR analysis. Moreover, no signal was observed in the no-template control in qPCR reactions for each primer pair, which
Statistical analysis: Three repetitions were performed to determine each value and standard deviations were calculated. Data were presented as means \pm SDs, and the statistical significance was determined by Student's $t$ test. suggested that there were no genomic DNA contaminants and primer dimers in the reaction mixture (Fig. 2. Suppl.). Additionally, standard curves were plotted using threefold serially diluted cDNA for the pooled samples. For all the primer pairs, the linear correlation coefficient $\left(r^{2}\right)$ was greater than 0.986 , while the specific PCR amplification efficiency (E) ranged from 92.7 to $108.6 \%$, respectively (Table 1 Suppl.).

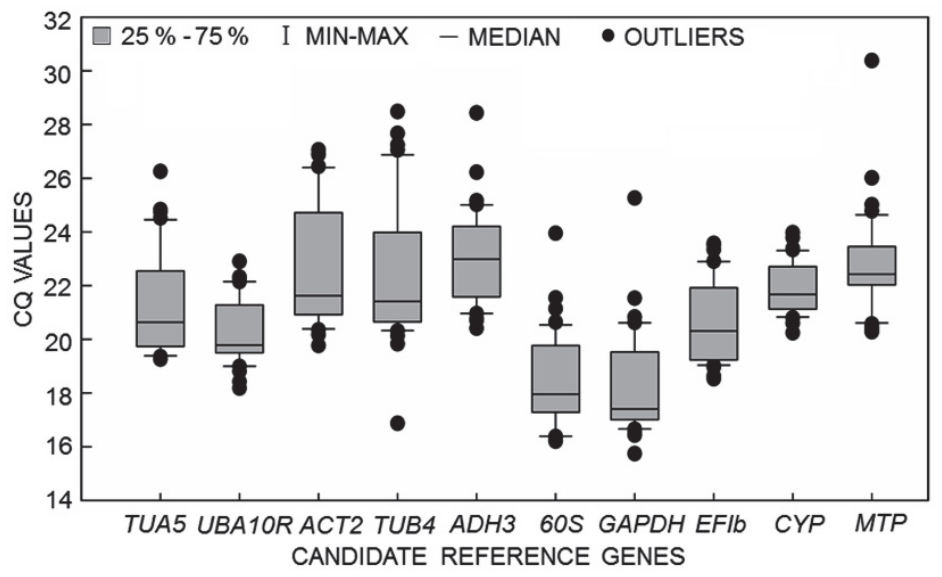

Fig. 1. Box-whisker plot of expression of ten candidate reference genes obtained from all the tested peanut samples. The figure was drawn by Sigmaplot 10.0 software according to the raw Cq values obtained from RT-qPCR. A lower Cq value represents more stable expression. The box represents the first $(25 \%)$ and the third $(75 \%)$ quartiles, whiskers depict the maximum and minimum values, while the line across the box and the black dots outside the box signifies the median and outlier values, respectively.

The $\mathrm{Cq}$ values of all the candidate reference genes varied from 15.74 to 31.38 , with most ranging from 18.63 to 25.01 (Fig. 1). The wide range of expressions reflected the lack of consistency and the necessity for normalization under stress responses. Among the ten tested genes, $U B Q 10 R, 60 S$, and GAPDH showed relatively low $\mathrm{Cq}$ values, indicating that they exhibited high stability. The $A D H 3$ showed the lowest stability (the highest mean $\mathrm{Cq}$ value of 22.86), followed by MTP with a mean Cq of 22.43 .

Using the geNorm, the M values of the candidate genes were calculated and ranked from the lowest to the highest (Fig. 2). The lowest $M$ value belongs to the most stable gene. Taking into account all the samples, $U B Q 10 R$ and $E F 1 b$ had the lowest M values (0.59), while $T U B 4$ had the highest $\mathrm{M}$ value (1.61) (Fig. $2 A$ ), indicating that $U B Q 10 R$ and $E F 1 b$ were both the most stable reference genes and TUB4 the least stable reference gene. Except for root tip and stem tissue of 99-1507, UBQ10R was the most stably expressed gene in all the tissues. $A C T 2 / 60 S(\mathrm{M}=0.24)$ was the most suitable gene pair for root tip and stem tissues of 99-1507, whereas $C Y P / 60 S$ ranked the best individual reference genes in the stem tissue of 99-1507. However, the gene with the least stable expression was TUB4 in all tissues of 99-1507 and the leaf of $\mathrm{ZH}_{2}$, whereas MTP (M $=2.08)$ and $A C T 2(\mathrm{M}=0.60)$, respectively, ranked the least stable in root tip and stem tissues of $\mathrm{ZH}_{2}$ (Fig. $2 B-G$ ). The results also showed that the most stable genes had an $M$ value below the threshold of 1.5, suggesting that they were suitable for consideration as reference genes for peanut under Al stress. Another function of geNorm was the determination of the optimal number of reference genes using the $V_{n / n+1}$ value (Fig. 3 ). The $V_{2} / V_{3}$ value was below 0.15 in all tissues of 99-1507 and the stem tissue of $\mathrm{ZH}_{2}$, suggesting that two reference genes were sufficient 
for normalization, but that three reference genes were necessary for the root tip and leaf tissues of $\mathrm{ZH}_{2}$. For the entire sample dataset, geNorm recommended six reference genes for normalization, because the six most stable genes had no marked effect on the normalization factor $\left(\mathrm{V}_{6} / \mathrm{V}_{7}=0.146\right)$.

The evaluation of reference genes for RT-qPCR normalization was further analyzed by Normfinder (Table 2 Suppl.). Intra- and inter-group variation was used to analyze all the samples and to subsequently calculate the $\mathrm{M}$ value to estimate stability. Similar to the geNorm method, the lower the $\mathrm{M}$ value, the more stable the gene is. $U B Q 10 R$ exhibited the most stable expression, with the lowest $M$ value in all the samples, ranking from 0.101 to
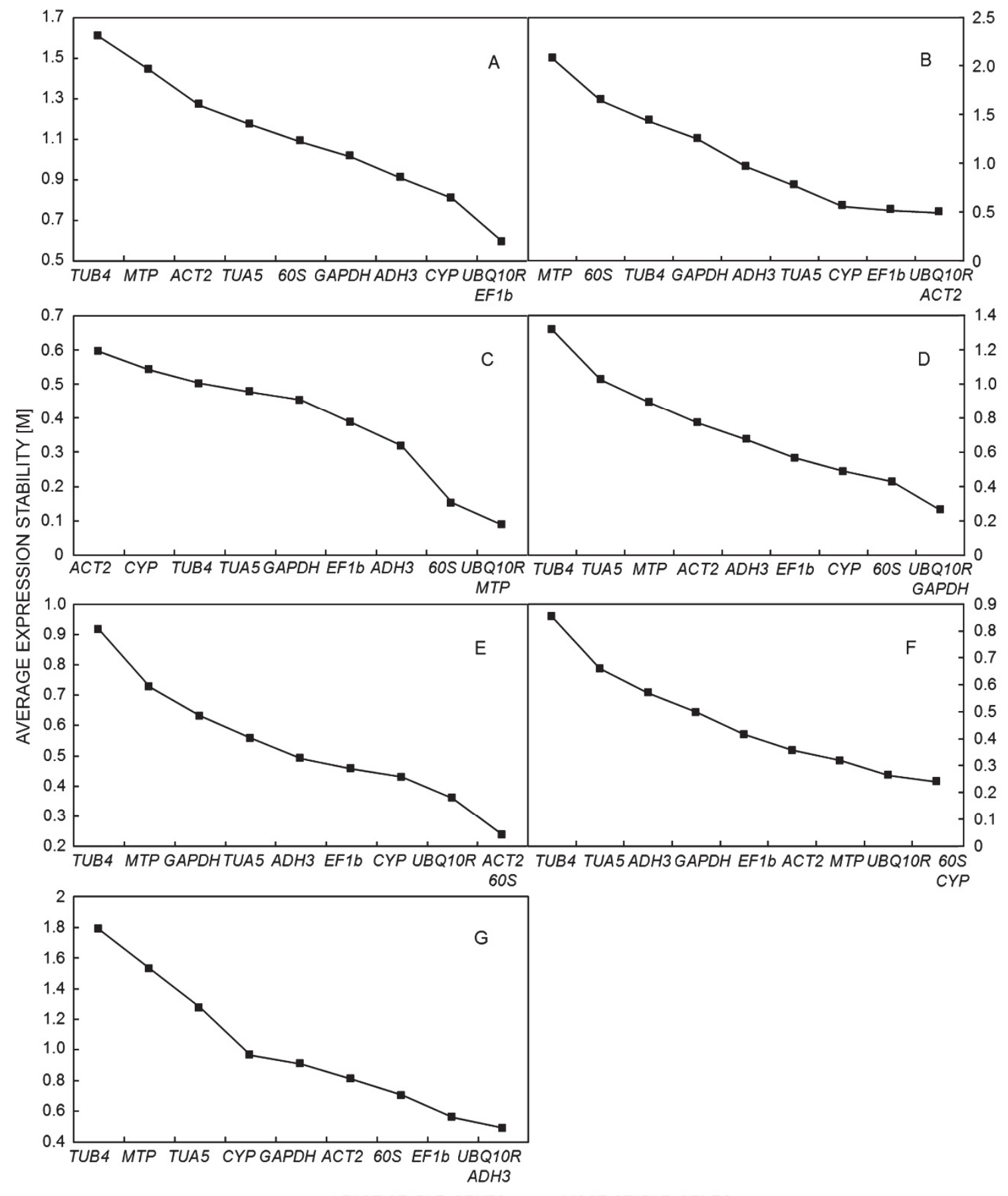

$<$ :...: LEAST STABLE GENES

Fig. 2. Gene average expression stability $(\mathrm{M})$ and ranking of potential reference genes in different tissues calculated by geNorm. A lower $\mathrm{M}$ value represents more stable expression analyzed in different sets, including all samples $(A)$, roots of $\mathrm{ZH}_{2}(B), \mathrm{stems}_{\mathrm{s}} \mathrm{ZH}_{2}$ $(C)$, leaves of $\mathrm{ZH}_{2}(D)$, roots of 99-1507 $(E)$, stems of 99-1507 $(F)$, and leaves of 99-1507 $(G)$. 
0.278. UBAQ10R and $A C T 2$ were recommended to be the best reference combination for all the samples, having an $\mathrm{M}$ value of 0.216 . TUB4 showed the lowest stability in all the samples expect for the root tip tissue of $\mathrm{ZH}_{2}$, where $M T P, 60 S$ and TUB4 showed the most variation.

The results from the Normfinder were largely consistent with those from the geNorm with regard to the normalization of a single candidate gene. However, the results from the BestKeeper, which mainly determined stability in terms of SD, were slightly different. $C Y P$ and $U B Q 10 R$ were highlighted as the best stable reference genes in the root tissue of $\mathrm{ZH}_{2}$ and the leaf tissue of 99-1507, respectively, while TUA5 was identified as the most suitable one for other samples (Table 3 Suppl.). The TUB4 had unstable expression in all the samples, as identified by the various software programs.

The geometric mean of each candidate gene from the three different program algorithms was calculated by the RefFinder in order to obtain more reliable and consistent results (Table 4 Suppl.). Based on the geometric mean, the ranking (in the orders of decreasing stability) of the ten candidate genes for the entire sample was $U B Q 10 R>$ $E F 1 b>C Y P=T U A 5>A C T 2>A D H 3>G A P D H>6 O S>$ $M T P>T U B 4$. For the root, stem, and leaf tissues of $\mathrm{ZH}_{2}$, $U B Q 10 R$ and TUA5, UBQ10R and EF1b, and UBQ10R and $E F 1 b$, respectively, were suggested to be the most

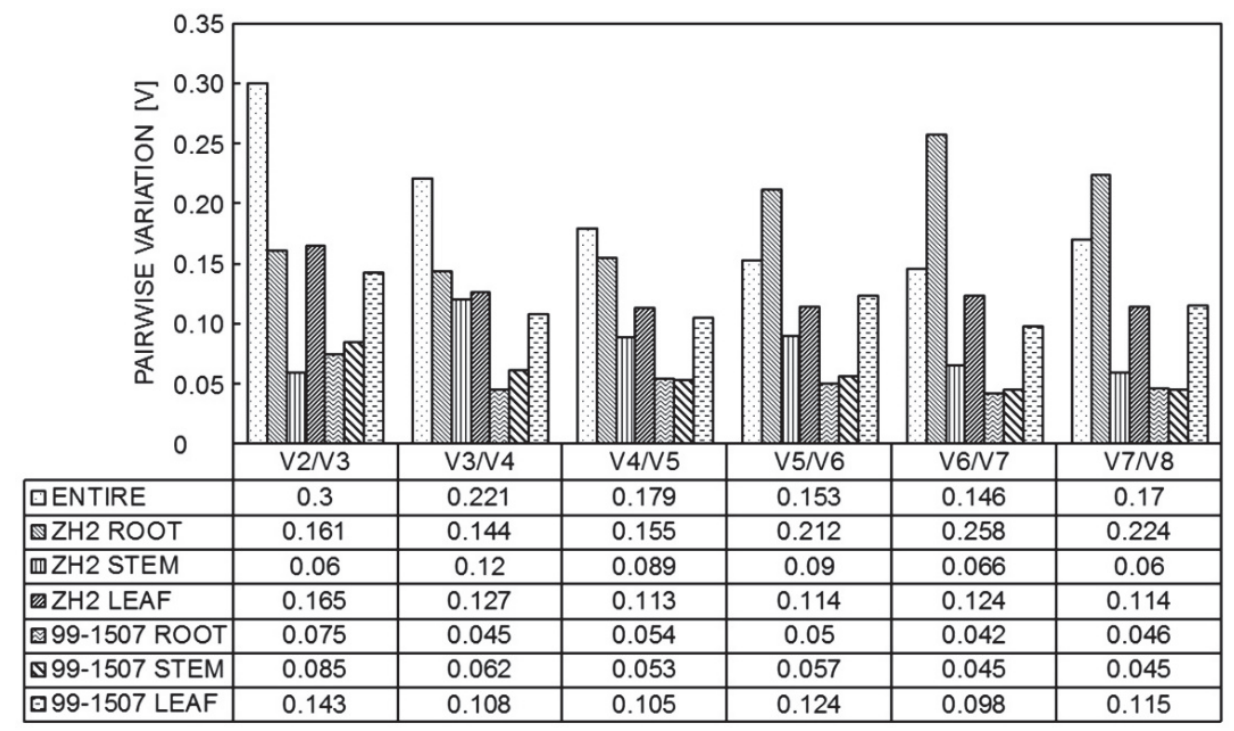

Fig. 3. Pairwise variation of the ten candidate genes was predicted by geNorm. The pairwise variation $\left(\mathrm{V}_{\mathrm{n}} / \mathrm{V}_{\mathrm{n}+1}\right)$ was calculated between the normalization factors $\mathrm{NF}_{\mathrm{n}}$ and $\mathrm{NF}_{\mathrm{n}+1}$, with a recommended cut off threshold of 0.15 .

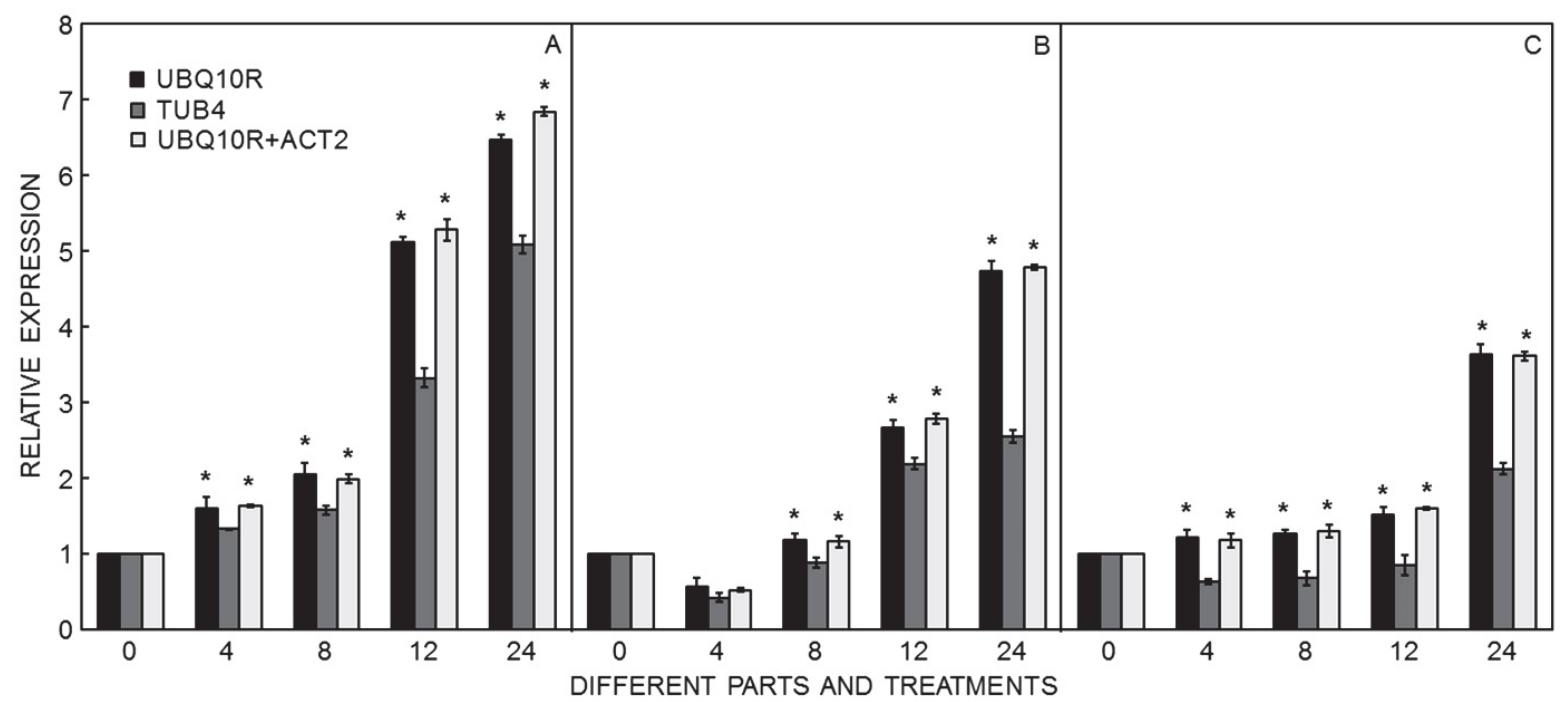

Fig. 4. Relative expression of the $A h M C 1$ gene during Al-induced PCD determined in roots $(A)$, stems $(B)$, and leaves $(C)$ of $\mathrm{cv} \mathrm{ZH}$ treated with $100 \mu \mathrm{M} \mathrm{AlCl}_{3}$ for $0,4,8,12$, and $24 \mathrm{~h}$, respectively. We selected the most stable reference gene $U B Q 10 R$ and the worst one TUB4 identificated by RefFinder, and the best combination [UBQ10R $+A C T 2]$ identified by Normfinder. Means $\pm \mathrm{SDs}, n=3$, * - significant difference at $P<0.05$ (Student's $t$-test). 
stable reference genes. Meanwhile, for the root, stem, and leaf tissues of 99-1507, UBQ10R and $C Y P, U B Q 10 R$ and $A C T 2$, and $U B Q 10 R$ and $A D H 3$, respectively, were highlighted as the reference genes exhibiting the least variation in expression.

To reinforce the results obtained from the software, the relative expression of $A h M C 1$ gene was analyzed, using the most stable reference gene $U B Q 10 R$ and the least stable gene TUB4 determined by RefFinder algorithms, and the best combination [UBQ10R+ACT2] identificated by Normfinder algorithms. The AhMC1 gene from peanut, belonging to the caspase-like protease family, have a positive role in Al-induced PCD (Yao et al. unpublished data). The AhMCl expression pattern and transcript abundance were similar with the candidate reference gene $U B Q 10 R$ individually and in the [UBQ10R+ACT2] combination, having no significant difference. On the contrary, normalization with the least stable one (TUB4) demonstrated underestimation of $A h M C 1$ expression in Al-treated samples, which was significantly lower than after normalization with $U B Q 10 R$ and [UBQ10R+ACT2] $(P<0.05)$ (Fig. 4).

\section{Discussion}

Al toxicity is one of the major limiting factors for peanut production due to induction of root tip PCD. In our previous studies, Al induced $\mathrm{PCD}$ rapidly in the Al-sensitive cultivar $\mathrm{ZH}_{2}$ and more slowly in the Al-tolerant cultivar 99-1507. However, the mechanism of Al-induced PCD is still unclear (Huang et al. 2014b). The transcriptomics data from these two cultivars during Al-induced PCD showed that there were many up- or down-regulated genes after $\mathrm{Al}$ treatment (Yao et al. unpublished data). Understanding the temporal and spatial expression patterns of these genes would be invaluable in clarifying the mechanism of Al-induced PCD.

Several researchers (Yael and Ran 2010, Morgante et al. 2011, Reddy et al. 2013) have reported different patterns of reference genes at different developmental stages, or various experimental conditions in peanut. Differential stability of reference genes in different tissues has also been reported in other plants (Lin et al. 2013, Imai et al. 2014, Hashemi et al. 2016, Hou et al. 2017 ). Some reference genes, like $A D H 3$ and $T U A 5$, have previously been highlighted as the most stable genes in peanut, but they are not suitable to all experimental sets. Here, the expression analysis of ten candidate reference genes in a pool of peanut samples containing three tissues, two cultivars, and Al-stress, showed that $U B Q 10 R$ was the most suitable reference gene for normalization. Except for $U B Q 10 R$, the top-ranked stable reference genes in individual samples were quite different, such as $E F 1 b$ in the stem and leaf of $\mathrm{ZH}_{2}, A C T 2$ in the stem of 99-1507, TUA5 in the root of $\mathrm{ZH}_{2}$ and so on (Table 4 Suppl.). This illustrated necessity for validating reference genes in peanut for specific experimental conditions. Prior to conducting a study on gene expression associated with Al-induced PCD in peanut, it was vital to carry out detailed research on the stability of candidate reference genes. RefFinder ranked the candidate genes on the basis of output from the different algorithms, including geNorm, NormFinder and BestKeeper, when the results of algorithms were different (Freitas et al. 2017). Volland et al. (2016) suggested the use of the RefFinder tool as an alternative to a global ranking tool to improve normali- zation of reference genes. In the present study, the ranking orders of reference genes generated by geNorm and NormFinder were different, but their selection for the best was nearly uniform, while the result from BestKeeper was quite different. RefFinder was needed to rank the candidate genes on the basis of outputs from three different algorithms. The results of RefFinder algorithms further recommended $U B Q 10 R$ and $E F 1 b$ to be the most suitable reference genes for normalization during gene expression studies in Al-induced PCD. In previous studies, $U B Q$ had also been used as the reference gene for the analysis of RT-qPCR in banana fruit under stress (chilling, high temperature, and pathogen) (Chen et al. 2011), cotton under salinity and drought (Wang et al. 2013), perennial ryegrass under different abiotic stresses (Yan et al. 2014), and Sapium sebiferum affected by sucrose (Chen et al. 2017), indicating that $U B Q$ is related to stress response. Elongation factor $E F 1$ has been reported to be the most stable gene in terms of expression in peanut under cold treatment (Chen et al. 2011, Chi et al. 2012), and in switch grass (Huang et al. 2014a) and African oil palm (Xia et al. 2014) under abiotic stresses. The expression stability of genes is related to the function they play in biological metabolic pathways (Wang et al. 2017). Ubiquitin is a small protein existing in all eukaryotes with a conserved sequence and structure, which plays important roles in controlling cellular signal transduction (Zhang 2011). $E F 1 b$ is mainly in charge of the initial steps of protein synthesis, and also exhibits conserved sequences. Acevedo et al. (2018) reported that elongation factor 1-alpha + tubulin alpha chain $(E F 1 a+\alpha-T u b)$ is one of the most suitable genes to compute a normalization factor in Ilex paraguariensis leaves during drought. The central and ubiquitous roles of these genes are likely to be the reasons which $U B Q 10 R$ and EF1b exhibit stable expression in many species.

On the other hand, TUB4 was the most unstable reference gene as ranked by all four algorithms, but it is interesting to note that $T U B$ was identified to be one of the best reference genes for gene expression in maize under different abiotic stresses (Lin et al. 2014) and in 
Amorphophallus under waterlogging (Wang et al. 2017). Nevertheless, it was not surprising that very different results were obtained in our study, reinforcing the necessity of reference gene normalization for expression studies prior to RT-qPCR analysis.

For a long time, GADPH was frequently selected to be the appropriate reference gene in many studies without validation (Gutierrez et al. 2008a,b). However, GADPH expression was found to be generally less stable under abiotic stress in our study, similar to the results from Chrysanthemum (Gu et al. 2011), maize (Galli et al. 2013), strawberry (Galli et al. 2015), lettuce (Borowski et al. 2014) and Setaria viridis (Martins et al. 2016). Hence, we do not recommend GADPH as a reference gene in peanut under Al-induced stress.

A single reference gene can be used if it shows expressional consistency in different experimental treatments, but in several instances the use of more than one reference gene as normalizers rather than only one proved to be more reliable (Vandesompele et al. 2002). According to the results obtained by geNorm, more than six genes for entire samples and at least two genes for different tissues should be hypothetically used to normalize the RT-qPCR experiments (Fig. 3). The NormFinder program suggested the combination of $[U B Q 10 R+A C T 2]$ as the most suitable gene combination

\section{References}

Acevedo, R.M., Avico, E.H., Ruiz, O.A., Sansberro, P.A.: Assessment of reference genes for real-time quantitative PCR normalization in Ilex paraguariensis leaves during drought. - Biol. Plant. 62: 89-96, 2018.

Andersen, C.L., Jensen, J.L., Ørntoft, T.F.: Normalization of real-time quantitative reverse transcription-PCR data: a model-based variance estimation approach to identify genes suited for normalization, applied to bladder and colon cancer data sets. - Cancer Res. 64: 5245-5250, 2004.

Borowski, J.M., Galli, V., Messias, R.D.S., Perin, E.C., Buss, J.H., Silva, S.D.D.A.E., Rombaldi, C.V.: Selection of candidate reference genes for real-time PCR studies in lettuce under abiotic stresses. - Planta 239: 1187-1200, 2014.

Castro-Quezada, P., Aarrouf, J., Claverie, M., Favery, B., Mugniéry, D., Lefebvre, V., Caromel, B.: Identification of reference genes for normalizing RNA expression in potato roots infected with cyst nematodes. - Plant mol. Biol. Rep. 31: 936-945, 2013.

Chen, L., Zhong, H.Y., Kuang, J.F., Li, J.G., Lu, W.J., Chen, J.Y.: Validation of reference genes for RT-qPCR studies of gene expression in banana fruit under different experimental conditions. - Planta 234: 377-390, 2011.

Chen, X., Mao, Y., Huang, S., Ni, J., Lu, W., Hou, J., Wang, M., Zhao, W., Li, M., Wang, Q., Wu, L.: Selection of suitable reference genes for quantitative real-time PCR in Sapium sebiferum. - Front. Plant Sci. 8: 637-648, 2017.

Chi, X., Hu, R., Yang, Q., Zhang, X., Pan, L., Chen, N., Chen, M., Yang, Z., Wang, T., He, Y., Yu, S.: Validation of reference genes for gene expression studies in peanut by
(Table 2 Suppl). To test this proposal, we analyzed the expression of the $A h M C 1$ gene by using $U B Q 10 R$ (the most stable gene), TUB4 (the least stable gene) and [UBQ10R + ACT2] (the most suitable combination) in the $\mathrm{ZH}_{2}$ cultivar under $\mathrm{Al}$ stress. The expression profiles of the $A h M C 1$ gene were similar when normalized by $U B Q 10 R$ individually and in the combination of [UBQ1OR +ACT2] (Fig. 4), suggesting that almost no significant difference was found between the best reference gene and the best gene combination in RT-qPCR normalization. However, there was a significant difference in target gene expression with the least stable gene TUB4 as reference gene. Therefore, the most stable normalizers selected by RefFinder and the most stable combination opted by Normfinder yielded similar expression profiles of the AhMCl gene, confirming the significance of validating the stability of reference gene prior to its application in RT-qPCR experiments.

This is the first study to validate a set of ten potential reference genes for RT-qPCR in peanut under Al-induced PCD. Our findings suggested that reference genes should be pre-evaluated for their expression stability in different experimental conditions. The selection of the reference genes recommended here would ensure accuracy in RT-qPCR results, which would also provide precious data for research stress tolerance mechanisms in peanut.

quantitative real-time RT-PCR. - Mol. Genet. Genomics 287: 167-176, 2012.

Freitas, N.C., Barreto, H.G., Fernandes-Brum, C.N., Moreira, R.O., Chalfun, A., Jr., Paiva, L.V.: Validationof reference genes for qPCR analysis of Coffea arabica L. somatic embryogenesis-related tissues. - Plant Cell Tissue Organ Cult. 128: 663-678, 2017.

Galeano, E., Vasconcelos, T.S., Ramiro, D.A., Martin, V.D.F.D., Carrer, H.: Identification and validation of quantitative real-time reverse transcription PCR reference genes for gene expression analysis in teak ( Tectona grandis L.). - BMC Res. Notes 7: 464, 2014.

Galli, V., Borowski, J.M., Perin, E.C., Messias, R.S., Labonde, J., Pereira, I.S., Silva, S.D., Rombaldi, C.V.: Validation of reference genes for accurate normalization of gene expression for real time-quantitative PCR in strawberry fruits using different cultivars and osmotic stresses. - Gene 554: 205-214, 2015.

Galli, V., Da Silva Messias, R., Dos Anjos e Silva, S.D., Rombaldi, C.V.: Selection of reliable reference genes for quantitative real-time polymerase chain reaction studies in maize grains. - Plant Cell Rep. 32: 1869-1877, 2013.

Gu, C., Chen, S., Liu, Z., Shan, H., Luo, H., Guan, Z., Chen, F.: Reference gene selection for quantitative real-time PCR in Chrysanthemum subjected to biotic and abiotic stress. - Mol. Biotechnol. 49: 192-197, 2011.

Gutierrez, L., Mauriat, M., Guénin, S., Pelloux, J., Lefebvre, J., Louvet, R.: The lack of a systematic validation of reference genes: a serious pitfall undervalued in reverse transcription 
polymerase chain reaction (RT-PCR) analysis in plants. Plant Biotechnol. J. 6: 609-618, 2008a.

Gutierrez, L., Mauriat, M., Pelloux, J., Bellini, C., Van Wuytswinkel, O.: Towards a systematic validation of references in real-time RT-PCR. - Plant Cell 20: 1734-1735, 2008 b.

Hashemi, S.H., Nematzadeh, G., Ahmadian, G., Yamchi, A., Kuhlmann, M.: Identification and validation of Aeluropus littoralis reference genes for quantitative real-time PCR normalization. - J. biol Res. 23: 18, 2016.

Hou, F., Li, S., Wang, J., Kang, X., Weng, Y., Xing, G. Identification and validation of reference genes for quantitative real-time PCR studies in long yellow daylily, Hemerocallis citrina Borani. - Plos ONE 12: e 0174933, 2017.

Huang, L.K., Yan, H.D., Jiang, X.M., Zhang, X.Q., Zhang, Y.W., Xiu, H., Yu, Z., Miao, J.M., Xu, B., Frazier, T.: Evaluation of candidate reference genes for normalization of quantitative RT-PCR in switchgrass under various abiotic stress conditions. - Bioenergy Res. 7: 1201-1211, 2014a.

Huang, W.J., Oo, T.L., He, H.Y., Wang, A.Q., Zhan, J., Li, C.Z., Wei, S.Q., He, L.F.: Aluminum induces rapidly mitochondria-dependent programmed cell death in A1sensitive peanut root tips. - Bot. Stud. 55: 67-79, $2014 \mathrm{~b}$.

Imai, T., Ubi, B.E., Saito, T., Moriguchi, T.: Evaluation of reference genes for accurate normalization of gene expression for real time-quantitative PCR in Pyrus pyrifolia using different tissue samples and seasonal conditions. Plos ONE 9: e86492, 2014.

Jiao, J., Zhou, B., Zhu, X., Gao, Z., Liang, Y.: Fusaric acid induction of programmed cell death modulated through nitric oxide signalling in tobacco suspension cells. - Planta 238: 727-737, 2013.

Kim, B.R., Nam, H.Y., Kim, S.U., Kim, S.I., Chang, Y.J.: Normalization of reverse transcription quantitative-PCR with housekeeping genes in rice. - Biotechnol. Lett. 25: 1869-1872, 2003

Kulcheski, F.R., Marcelino-Guimaraes, F.C., Nepomuceno, A.L., Abdelnoor, R.V., Margis, R.: The use of microRNAs as reference genes for quantitative polymerase chain reaction in soybean. - Anal. Biochem.406: 185-192, 2010.

Kundu, A., Patel, A., Pal, A.: Defining reference genes for qPCR normalization to study biotic and abiotic stress responses in Vigna mungo. - Plant Cell Rep. 32: 1647-1658, 2013.

Li, Q., Fan, C.M., Zhang, X.M., Fu, Y.F.: Validation of reference genes for real-time quantitative PCR normalization in soybean developmental and germinating seeds. - Plant Cell Rep. 31: 1789-1798, 2012.

Lin, L., Han, X., Chen, Y., Wu, Q., Wang, Y.: Identification of appropriate reference genes for normalizing transcript expression by quantitative real-time PCR in Litsea cubeba. - Mol. Gene.t Genomics.288: 727-737, 2013.

Lin, Y., Zhang, C., Lan, H., Gao, S., Liu, H., Liu, J., Cao, M., Pan, G., Rong, T., Zhang, S.: Validation of potential reference genes for qPCR in maize across abiotic stresses, hormone treatments, and tissue types. - Plos ONE 9: e95445, 2014.

Ling, H., Wu, Q., Guo, J., Xu, L., Que, Y.: Comprehensive selection of reference genes for gene expression normalization in sugarcane by real time quantitative RTPCR. - Plos ONE 9: e97469, 2014.
Martins, P.K., Mafra, V., Souza, W.R.D., Ribeiro, A.P., Vinecky, F., Basso, M.F., Kobayashi, A.K., Molinari, H.B.C.: Selection of reliable reference genes for RT-qPCR analysis during developmental stages and abiotic stress in Setaria viridis. - Sci. Rep. 6: 28348-28358, 2016.

Mehdi, K.K., Van, B.E.: A critique of widely used normalization software tools and an alternative method to identify reliable reference genes in red clover (Trifolium pratense L.). - Planta 236: 1381-1393, 2012.

Morgante, C.V., Guimarães, P.M., Martins, A.C.Q., Araújo, A.C.G., Leal-Bertioli, S.C.M., Bertioli, D.J., Brasileiro, A.C.M.: Reference genes for quantitative reverse transcription-polymerase chain reaction expression studies in wild and cultivated peanut. - BMC Res. Notes 4: 339, 2011.

Nolan, T., Hands, R.E., Bustin, S.A.: Quantification of mRNA using real-time RT-PCR. - Natur. Protocols 1: 1559-1582, 2006.

Pan, J.W., Zhu, M.Y., Chen, H.: Aluminum-induced cell death in root-tip cells of barley. - Environ. exp. Bot. 46: 71-79, 2001.

Patankar, H., M Assaha, D.V., Al-Yahyai, R., Sunkar, R., Yaish, M.W.: Identification of reference genes for quantitative real-time PCR in date palm (Phoenix dactylifera L.) subjected to drought and salinity. - Plos ONE 11: e01662160166216, 2016.

Pfaffl, M.W., Tichopad, A., Prgomet, C., Neuvians, T.P.: Determination of stable housekeeping genes, differentially regulated target genes and sample integrity: BestKeeperexcel-based tool using pair-wise correlations. - Biotechnol. Lett. 26: 509-515, 2004.

Reddy, D.S., Bhatnagar-Mathur, P., Cindhuri, K.S., Sharma, K.K.: Evaluation and validation of reference genes for normalization of quantitative real-time PCR based gene expression studies in peanut. - Plos ONE 8: e78555, 2013.

Stephenson, F.H.: The real-time polymerase chain reaction (RTPCR). - In: Stephenson, F.H. (ed.): Calculations for Molecular Biology and Biotechnology. Vol. 9. Pp 211-311. Academic Press, New York - London 2010.

Sugden, D., Winter, P.D. (ed.): Quantification of mRNA Using Real Time RT-PCR. - Humana Press,New Jersey 2008.

Vandesompele, J., Preter, K.D., Pattyn, F., Poppe, B., Roy, N.V., Paepe, A.D., Speleman, F.: Accurate normalization of real-time quantitative RT-PCR data by geometric averaging of multiple internal control genes. - Genome Biol. 3: research0034.1, 2002.

Van Guilder, H.D., Vrana, K.E., Freeman, W.M.: Twenty-five years of quantitative PCR for gene expression analysis. Biotechniques 44: 619-626, 2008.

Volland, M., Blasco, J., Hampel, M.: Validation of reference genes for RT-qPCR in marine bivalve ecotoxicology: systematic review and case study using copper treated primary Ruditapes philippinarum hemocytes. - Aquat. Toxicol. 185: 86-94, 2016.

Wan, C.Y., Wilkins, T.A.: A modified hot borate method significantly enhances the yield of high-quality RNA from cotton (Gossypium hirsutum L.). - Anal Biochem. 223: 7 12, 1994.

Wang, K., Niu, Y., Wang, Q., Liu, H., Jin, Y., Zhang, S.: Cloning and evaluation of reference genes for quantitative real-time PCR analysis in Amorphophallus. - Peer J. 5: e3260, 2017. 
Wang, M., Wang, Q., Zhang, B.: Evaluation and selection of reliable reference genes for gene expression under abiotic stress in cotton (Gossypium hirsutum L.). - Gene 530: 44$50,2013$.

Xia, W., Mason, A.S., Xiao, Y., Liu, Z., Yang, Y.D., Lei, X.T., Wu, X.M., Ma, Z.L., Peng, M.: Analysis of multiple transcriptomes of the African oil palm (Elaeis guineensis) to identify reference genes for RT-qPCR. - J. Biotechnol. 184: 63-73, 2014.

Xiang, F., Yang, M.S., Pil, S., Hong, S.Y., Chung-Mo, P.: Exploring valid reference genes for gene expression studies in Brachypodium distachyon by real-time PCR. - BMC Plant Biol. 8: 112, 2008.

Xie, F., Sun, G., Stiller, J.W., Zhang, B.: Genome-wide functional analysis of the cotton transcriptome by creating an integrated EST database. - Plos ONE 6: e26980, 2011.

Yael, B., Ran, H.: Identification of suitable internal control genes for quantitative real-time PCR expression analyses in peanut (Arachis hypogaea). - Peanut Sci. 37: 12-19, 2010.

Yan, H.D., Jiang, X.M., Zhang, X.Q., Huang, L.K.: Reference genes for qRT-PCR in perennial ryegrass (Lolium perenne L.) under various abiotic stresses. - J. agr. Biotechnol.12:
1494-1501, 2014.

Yao, S., Huang, W., Pan, C., Zhan, J., He, L.: Caspase-like proteases regulate aluminum-induced programmed cell death in peanut. - Plant Cell Tissue Organ Cult. 127: 691703, 2016.

Zhan, J., Li, W., He, H.Y., Li, C.Z., He, L.F.: Mitochondrial alterations during Al-induced PCD in peanut root tips. Plant Physiol. Biochem. 75: 105-113, 2014.

Zhang, X.Y. (ed.): Functional Studies of Arabidopsis Polyubiquitin Genes UBQ3 and UBQ4. - National Taiwan Normal University, Taipei 2011.

Zhong, H.Y., Chen, J.W., Li, C.Q., Chen, L., Wu, J.Y., Chen, J.Y., Lu, W.J., Li, J.G.: Selection of reliable reference genes for expression studies by reverse transcription quantitative real-time PCR in litchi under different experimental conditions. - Plant Cell Rep. 30: 641-653, 2011.

Zsóri, K.S., Muszbek, L., Csiki, Z., Shemirani, A.H.: Validation of reference genes for the determination of platelet transcript level in healthy individuals and in patients with the history of myocardial infarction. - Int. J. mol. Sci. 14: 3456-3466, 2013. 